\title{
Evaluation of Dental Caries and Concomitant Infection Based on the Ratio of Cariogenic Bacteria using Plaque Samples in Adults
}

Hiroya Gotouda $^{1^{*}}$, Noriko Shinozaki-Kuwahara ${ }^{2}$, Chieko Taguchi ${ }^{1}$, Mitsuhiro Ohta ${ }^{3}$, Michiharu Shimosaka ${ }^{4}$, Takanori Ito ${ }^{3}$, Koichi Hiratsuka $^{5}$, Tomoko KuritaOchiai $^{2}$ and Ikuo Nasu ${ }^{1}$

${ }^{1}$ Department of Preventive and Public Oral Health. Nihon University School of Dentistry at Matsudo, Matsudo, Chiba 271-8587, Japan

${ }^{2}$ Department of Microbiology and Immunology, Nihon University School of Dentistry at Matsudo, Matsudo, Chiba 271-8587, Japan

${ }^{3}$ Department of Oral Diagnostics, Nihon University School of Dentistry at Matsudo, Matsudo, Chiba 271-8587, Japan

${ }^{4}$ Department of Anesthesiology, Nihon University School of Dentistry at Matsudo, Matsudo, Chiba 271-8587, Japan

${ }^{5}$ Department of Biochemistry and Molecular Biology, Nihon University School of Dentistry at Matsudo, Matsudo, Chiba 271-8587, Japan

*Corresponding author: Hiroya Gotouda, D.D.S, PhD, Department of Preventive and Public Oral Health, Nihon University School of Dentistry at Matsudo, Matsudo, Chiba 271-8587, Japan, E-mail: gotouda.hiroya@nihon-u.ac.jp

Received date: October 09, 2017; Accepted date: October 23, 2017; Published date: October 30, 2017

Copyright: $\odot 2017$ Gotouda $\mathrm{H}$, et al. This is an open-access article distributed under the terms of the Creative Commons Attribution License, which permits unrestricted use, distribution, and reproduction in any medium, provided the original author and source are credited.

\begin{abstract}
Background: The aim of this study was to evaluate the association between dental caries and concomitant infections of Streptococcus mutans (S. mutans) and Streptococcus sobrinus (S. sobrinus) according to the ratio of cariogenic bacteria in plaque samples from adults to obtain basic data to develop clinical and chairside culture assay (kits) for assessment of dental caries risk.
\end{abstract}

Methods and Materials: Plaque samples from 192 adult volunteers (age range, 20-28 years) were obtained using sterile toothbrushes. Caries history and the number of decayed, missing, and filled (DMF) teeth were determined. Subjects were divided into high- and low-risk groups according to the ratio of $S$. mutans to total streptococci (Sm/TS ratio) and the number of S. mutans.

Results: The numbers of S. mutans and S. sobrinus and the Sm/TS ratio were significantly greater in the cariesactive (CA) group than in the caries-free (CF) group. The number of bacteria and the Sm/TS ratio for the high-risk $S$. mutans plus detectable $S$. sobrinus subgroup was significantly greater in the CA group than in the CF group. High levels of $S$. mutans and $S$. sobrinus were significantly associated with dental caries. A significantly high caries risk was found between DMF teeth and the Sm/TS ratio for S. mutans when S. sobrinus was detected.

Conclusions: The number of dental caries was greater in adults with plaque samples concomitantly infected with high levels of $S$. mutans and $S$. sobrinus than in those with a single infection. These findings indicate the usefulness of analysis of cariogenic dental bacteria to detect adult populations with the severity of dental caries according to the $\mathrm{Sm} / \mathrm{TS}$ ratio using dental plaque samples.

Keywords: Concomitant infection; Dental caries; Toothbrush plaque samples; Cariogenic bacteria ratio; Public health; Epidemiology

\section{Introduction}

Total Streptococci can be roughly grouped into the following four main species: mutans streptococci (MS), Streptococcus mitis, Streptococcus salivarius, and Streptococcus sanguis, which accounts for the majority of bacteria found in the mouth [1]. Among species that are generally termed MS, seven cariogenic streptococcal species, namely Streptococcus mutans (S. mutans), Streptococcus sobrinus ( $S$. sobrinus), Streptococcus rattus, Streptococcus cricetus, Streptococcus downei, Streptococcus macacae, and Streptococcus ferus, are responsible for dental caries formation [2-7]. However, high caries activity has been shown to be more strongly associated with the prevalence of S. sobrinus than S. mutans [8-10]. Nonetheless, both species are considered important for the diagnosis and prevention of dental decay. Therefore, evaluation of the concomitant infection of S. mutans and S. sobrinus is necessary to assess the risk of dental caries with high precision.
Infants and children have been the subjects of most studies of dental caries formation [9-16], as reports of a single infection in adults $[17,18]$ are scarce and no study of plaque samples has investigated concomitant infections in adults. Plaque, which harbors cariogenic bacteria, is an ideal sample for the assessment of caries activity $[1,19]$. However, as it is difficult to quantify plaque samples, paraffin- or chewing gum-stimulated saliva is more commonly used for the assessment of dental caries risk [2,4,5]. Among such sampling methods, the processing of plaque samples collected by brushing (plaque suspension) allows for quantitation and simple adjustments [20]. Therefore, plaque may be a clinically useful sample. However, this past study has focused on the reproducibility and validity of plaque samples, while none has investigated dental caries and the concomitant infection. Assessment of cariogenic bacteria levels is often used to determine the number of cariogenic bacteria [1-5]. However, few studies have reported the utility of the ratio of MS to total streptococci (TS) (MS/TS ratio) or $S$. mutans to TS (Sm/TS ratio) as an alternative approach to evaluate cariogenic bacteria [20,21]. Although the validity and reproducibility of these methods have been evaluated, an index 
must have an association with dental caries to be a useful standard to assess the risk of dental caries [1-3]. To date, no study has evaluated the association of these ratios with dental caries.

It is difficult to obtain samples from children and elderly individuals with common methods that involve collection of saliva while chewing gum. Additionally, the use of plaque specimens is challenging owing to problems with quantitation and reproducibility, and it is much easier to obtain samples for quantitative plaque analysis by brushing. Simple commercially available culture kits have been used for clinical and chairside cultures, as well as clinical public health studies [1-3]. These kits can conveniently detect and estimate MS at chairside without the need for expensive equipment. However, these kits use saliva samples and only identify the seven bacterial strains of the MS group. Therefore, these kits cannot be used to specifically evaluate S. mutans, which is considered the most important pathogen in dental caries formation. An immunochromatographic assay that employs a monoclonal antibody to selectively detect $S$. mutans has been reported [2]. Although an immunohistochemistry kit was released commercially as an alternative to culture methods, it was later discontinued owing to imprecision and high cost. Molecular, biological, and epidemiological studies have demonstrated that $S$. sobrinus is a strong cariogenic species and infection of both S. mutans and S. sobrinus synergistically increases the risk of cariogenesis $[2,4,7,22]$. However, no simple assay (kit) is available for the easy detection of S. sobrinus.

The aim of this study was to evaluate the association between dental caries and concomitant infections of $S$. mutans and $S$. sobrinus according to the ratio of cariogenic bacteria from adults using plaque samples harvested from sterile toothbrushes. This pilot study obtained fundamental data for the development of a more precise clinical and chairside culture assay (kits) for assessment of dental caries risk, which employs quantitative plaque analysis and the $\mathrm{Sm} / \mathrm{TS}$ ratio, without the use polymerase chain reaction (PCR) or immunohistochemical analysis.

\section{Materials and Methods}

\section{Subjects and preparation of plaque simple}

A total of 192 adult volunteers (age range, 20-28 years) in good physical condition with good oral health participated in this crosssectional study conducted at the Nihon University School of Dentistry at Matsudo. Caries status was investigated and plaque samples were collected at this institution. The study protocol was approved by the Ethics Committee of the Nihon University School of Dentistry at Matsudo and all subjects signed an informed consent form after being informed about the aim of this study. Subjects with any systemic disease, using medications affecting salivary secretions, taking antibiotics were excluded from the study. The selected individuals were instructed not to eat/drink, use a mouth wash, or smoke $3 \mathrm{~h}$ prior to their appointment.Each subject was instructed to rinse the mouth, and then a large portion of plaque from all teeth was scraped off by vigorous brushing for $1 \mathrm{~min}$ using a sterile toothbrush, and was collected into a sterile bottle through a mouth rinse for 30 s with $5 \mathrm{ml}$ phosphate-buffered saline and used as brushing-plaque sample [20].

\section{Bacterial analysis}

Mitis Salivarius agar plate (MS agar; Difco Laboratories, Inc., Detroit, MI, USA) and agar plate of Gold, [23] was used to culture bacteria. Within $3 \mathrm{~h}$ after collection, the clinically isolated samples were dispersed by sonication ( $50 \mathrm{~W}, 20 \mathrm{~s})$ using an ultrasonic apparatus (5202 Type; Ohtake Works Co., Ltd., Tokyo, Japan), serially diluted with chilled brain-heart infusion (BHI) broth. Laboratory strains and clinically isolated strains of S. mutans and S. sobrinus were cultured in BHI broth and incubated and inoculated on selective media using a spiral plating system and colony counter (Model-D; Gunze Sangyo, Inc., Tokyo, Japan). After anaerobic culture for $48 \mathrm{~h}$, the number of TS colonies and MS colonies agar plates were counted, respectively. $S$. mutans and $S$. sobrinus were distinguished according to the colony morphology on agar plates, respectively. $S$. mutans and $S$. sobrinus on agar was determined by counting the number of respective colonies, and the ratio of S. mutans to total streptococci was designated as the Sm/TS ratio. A stereomicroscope was used to differentiate between $S$. mutans and $S$. sobrinus on the basis of the morphology of colonies formed with sucrose, the differences arising from how glucan is synthesized by the GTF enzymes formed outside or on the outer layer of the bacterial body about ratio of cariogenic bacteria. The Enzyme Linked Immunosorbent Assay (ELISA) was also used in cases where differentiation proved difficult [20,21].

\section{Investigation of caries status and classification of caries risk groups}

Caries status was determined according to the standards of the World Health Organization [24]. The number of decayed, missing, and filled (DMF) teeth was determined and recorded for each subject. The subjects were classified into either a high-risk or low-risk group according to the abundance of $\mathrm{S}$. mutans and the $\mathrm{Sm} / \mathrm{TS}$ ratio (in descending order).

\section{Statistical analysis}

The Mann-Whitney U test, $\chi 2$ test, and Fisher's exact probability test were used for comparisons between two groups. The Bonferroni test was used for comparisons among four groups. Descriptive statistics were assessed and all statistical analyses were performed using SPSS ver. 22.0 software (IBM Corp., Armonk, NY, USA). Data are presented as mean values \pm standard deviation (SD).A probability (p) value $<0.05$ was considered statistically significant.

\section{Results}

The detection rate of TS in all subjects $(n=192)$ was $100 \%$. The detection rates of S. mutans and S. sobrinus were $92.2 \%$ and $21.9 \%$, respectively $\left(\mathrm{p}<0.001, \chi^{2}\right.$ test, Figure 1$)$. The mean $( \pm$ standard deviation, SD) number of detected TS was $9.86 \pm 12.01 \times 10^{7} \mathrm{CFU} / \mathrm{mL}$. The mean $( \pm \mathrm{SD})$ numbers of detected $S$. mutans and $S$. sobrinus bacteria were $24.42 \pm 78.93$ and $2.84 \pm 19.79 \times 10^{5} \mathrm{CFU} / \mathrm{mL}$, respectively. The mean $\mathrm{Sm} / \mathrm{TS}$ ratio (\%) was $2.21 \pm 4.87 \%$. The mean number of DMF teeth in all subjects was $7.89 \pm 5.97$. There were significant differences in the numbers of DMF teeth in subjects which had both $S$. mutans and $S$. sobrinus $(11.07 \pm 4.78)$ and DMF in subject with just $S$. mutans $(7.40 \pm 5.90),(\mathrm{p}<0.01$, Mann-Whitney U test).Subjects with $\geq 15$ DMF teeth were included in the caries-active (CA) group ( $n=25)$ and those with no DMF teeth were included in the caries-free $(\mathrm{CF})$ group $(\mathrm{n}=24)$. There were significantly greater numbers of $S$. mutans and $S$. sobrinus in the CA group than the CF group $(\mathrm{p}<0.001$ and $\mathrm{p}<0.01)$, respectively. Additionally, the $\mathrm{Sm} / \mathrm{TS}$ ratio (\%) was significantly greater in the CA group $(\mathrm{p}<0.001$, MannWhitney U test, Table 1). 
Citation: Gotouda H, Shinozaki-Kuwahara N,Taguchi C, Ohta M, Shimosaka M, et al. (2017) Evaluation of Dental Caries and Concomitant Infection Based on the Ratio of Cariogenic Bacteria using Plaque Samples in Adults. Dentistry 7: 461. doi:10.4172/2161-1122.1000461

Page 3 of 5

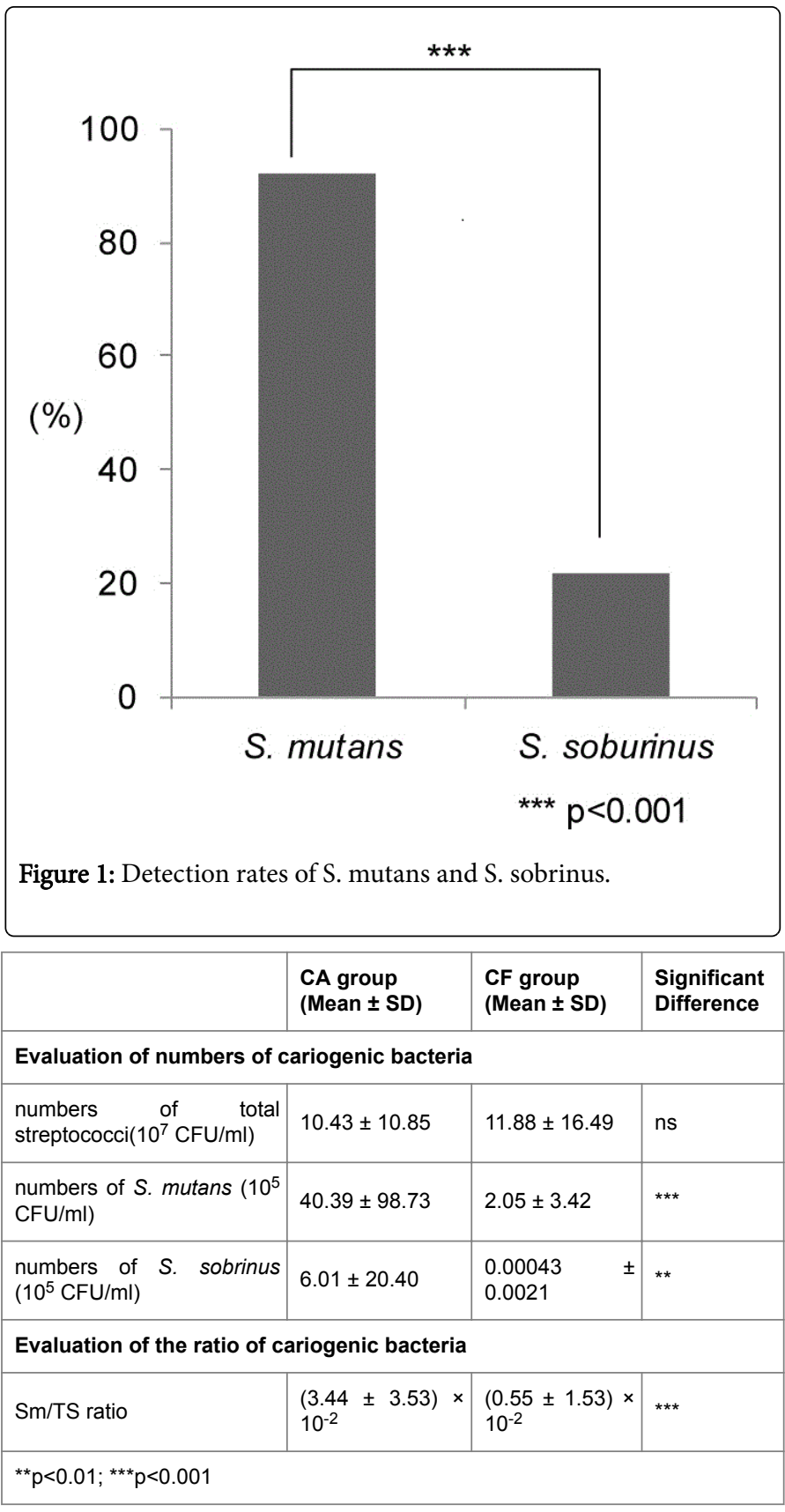

Table 1: Numbers and ratios of cariogenic bacteria in the CA and CF groups.

In the evaluation of the ratio of cariogenic bacteria, there were significant differences in between the CA and CF groups in the rates of a high-risk $\mathrm{Sm} / \mathrm{TS}$ ratio plus detectable $S$. sobrinus (Ratios-4G) $(32.0 \%$ vs. $0.0 \%$, respectively, $\mathrm{p}<0.01)$, the rates of a high-risk $\mathrm{Sm} / \mathrm{TS}$ ratio plus non-detectable S. sobrinus( Ratios-3G )(60.0\% vs. $20.8 \%$, respectively, $\mathrm{p}<0.01)$, and the rates of a low-risk Sm/TS ratio plus non-detectable $S$. sobrinus(Ratios-1G) $(4.0 \%$ vs. $75.0 \%$, respectively, $\mathrm{p}<0.001)$. In the evaluation of the number of cariogenic bacteria, there were significant differences between the CA and CF groups in the rates of high-risk S. mutans plus detectable S. sobrinus (Numbers- $4 \mathrm{G})(32.0 \%$ vs. $4.2 \%$, respectively, $\mathrm{p}<0.05)$ and the rates of low-risk $\mathrm{S}$. mutans plus non- detectable S. sobrinus (Numbers-1G) $(20.0 \%$ vs. $75.0 \%$, respectively, $\mathrm{p}<0.001$, Fisher's exact probability test, Table 2 ).

There were also significant differences in the numbers of DMF teeth in Ratios-4G ( $\mathrm{n}=25)$, Ratios-3G $(\mathrm{n}=71)$, Ratios-2G) $(\mathrm{n}=17)$, and Ratios-1G $(\mathrm{n}=79)$ subgroups $(13.0 \pm 4.3,9.5 \pm 6.6,7.2 \pm 4.4$, and $5.0 \pm$ 4.4 , respectively, $\mathrm{p}<0.05, \mathrm{p}<0.01$, and $\mathrm{p}<0.001$, respectively, Bonferroni test, Figure 2), as well as in the numbers of DMF teeth in Numbers-4G $(\mathrm{n}=26)$, Numbers-3G $(\mathrm{n}=70)$, Numbers-2G $(\mathrm{n}=16)$, and Numbers-1G $(\mathrm{n}=80)$ subgroups $(12.4 \pm 4.9,8.5 \pm 6.1,7.8 \pm 4.3$, and $5.9 \pm 5.6$, respectively, $(\mathrm{p}<0.05$ and $\mathrm{p}<0.001$, respectively, Bonferroni test, Figure 3).

\begin{tabular}{|c|c|c|c|}
\hline Risk group & $\begin{array}{l}\text { CA group } \\
(\%)\end{array}$ & $\begin{array}{l}\text { CF group } \\
(\%)\end{array}$ & $\begin{array}{l}\text { Significan } \\
\text { ce }\end{array}$ \\
\hline \multicolumn{4}{|c|}{$\begin{array}{l}\text { Evaluation of Ratios of cariogenic } \\
\text { bacteria }\end{array}$} \\
\hline High S.m/TS + S.s & 32 & 0 & ** \\
\hline High S.m/TS + nonS.s & 60 & 20.8 & ** \\
\hline Low S.m/TS + S.s & 4 & 4.2 & ns \\
\hline Low S.m/TS + nonS.s & 4 & 75 & $* * *$ \\
\hline \multicolumn{4}{|c|}{$\begin{array}{l}\text { Evaluation of numbers of cariogenic } \\
\text { bacteria }\end{array}$} \\
\hline High S.m + S.s & 32 & 4.2 & * \\
\hline High S.m + nonS.s & 44 & 20.8 & ns \\
\hline Low S.m + S.s & 4 & 0 & ns \\
\hline Low S.m + nonS.s & 20 & 75 & $* * *$ \\
\hline
\end{tabular}

Table 2: Rates of high- and low-risk S. mutans and ration of high- and low-risk S. mutans/total streptococci along with detectable and nondetectable S. sobrinus in the CA and CF groups.

\section{Discussion}

Cariogenic bacteria are transmitted and attach to the surfaces of the teeth of children after tooth eruption mainly via bacterial transfer from the mouths of close relatives (especially mothers) [1,4,25]. S. mutans and S. sobrinus are known to inhabit the human mouth at particularly high rates and are associated with dental caries formation [2-5]. The detected frequency varies, but S. mutans is detected in more than $90 \%$ of mouths, while S. sobrinus is detected in about $20 \%-30 \%$ by the culture technique [22]. The proportions of S. mutans and S. sobrinus detected in the mouths of adults in the present study were $92.2 \%$ and $21.9 \%$, respectively, consistent with the proportions reported previously. In this study, for comparison with Simple culture kits utilized in clinical studies and at the chairside, these similar agar medium (culture medium) is used. According to this distribution, the assessment of the risk of dental caries is often solely based on the presence of $S$. sobrinus infection (irrespective of whether S. sobrinus is present), without performing bacterial count or ratio assessment $[1,7,22]$. The present study targeted S. mutans only to determine the ratio of cariogenic bacteria. 
Page 4 of 5

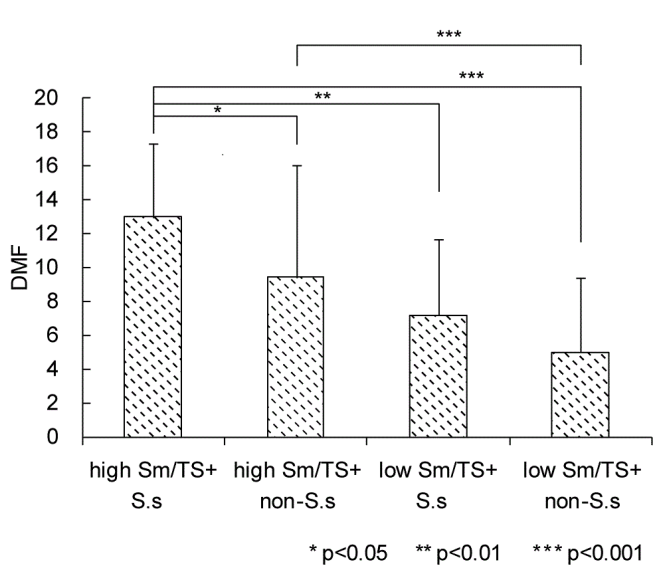

Figure 2: Numbers of decayed, missing, and filled (DMF) teeth in the high- and low-risk S. mutans/total streptococci ratio(Sm/TS) groups along with detectable and non-detectable S. sobrinus subgroups; Abbreviations: high Sm/TS, high-risk Sm/TS; low Sm/TS, low-risk Sm/TS; Ss, detectable S. sobrinus; non-Ss, nondetectable S. sobrinus.

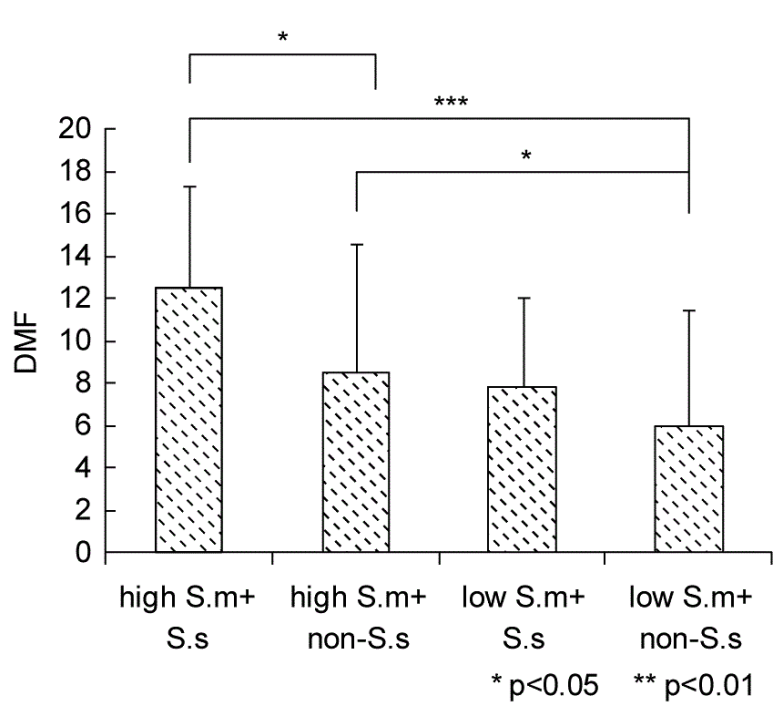

Figure 3: Numbers of decayed, missing, and filled (DMF) teeth in the high- and low-risk S. mutans numbers groups along with detectable and non-detectable $S$. sobrinus subgroups; Abbreviations: high Sm, high-risk S. mutans; low Sm, low-risk S. mutans; Ss, detectable S. sobrinus; non-Ss, non-detectable S. sobrinus.

Saliva stimulated by chewing has been used as a sample for the clinical detection and evaluation of the number of MS in the mouth, as it allows for quantitation [2]. Many dentists use simple culture kits to detect cariogenic bacteria with paraffin or chewing gum-stimulated saliva samples [2]. However, MS species are mainly found in plaque on the tooth surface [1]. Therefore, a bacterial count of plaque is required to clarify the caries-related activity of MS. A previous study that examined plaque collection methods to enable quantitation of bacteria revealed that plaque suspension after brushing teeth (brushing-plaque sample) is the best option [20]. In the present study, bacteriological test results were analyzed and evaluated using a culture method to quantitate $S$. sobrinus and $S$. mutans in brushing-plaque samples. In a previous study, the ratio of cariogenic bacteria obtained using a brushing-plaque sample was more reproducible than that obtained using a swabbing plaque sample [20]. The ratio of cariogenic bacteria in plaque was, on average, a few fold greater than that in saliva, indicating that plaque is a more useful sample than saliva [20]. However, the association between cariogenic bacteria in plaque with dental caries is a major requirement to consider a parameter appropriate for the assessment of caries activity [1-3]. Therefore, in the present study, the association between the ratio of cariogenic bacteria and dental caries in adults, and the usefulness of the brushing-plaque sample were assessed.

With regard to the associations of $S$. mutans and $S$. sobrinus with dental caries, $S$. mutans is isolated from dental caries more often than $S$. sobrinus, suggesting that $S$. mutans may be the major cariogenic Streptococcus species [26-29]. Furthermore, many epidemiological and in vitro studies have suggested that $S$. sobrinus is more strongly associated with the incidence of dental caries than $S$. mutans $[2,4,7,22]$. In the present study, the $\mathrm{Sm} / \mathrm{TS}$ ratio was significantly greater in the CA group than in the CF group. Additionally, the proportion of subjects with concomitant infections of high-risk $S$. mutans and $S$. sobrinus was significantly greater in the CA group, while the proportion with low-risk $S$. mutans and non-detectable $S$. sobrinus was greater in the CF group. Evaluations of the bacterial ratio and numbers found differences in the proportion of concomitant infections and the numbers of DMF teeth between the CA and CF groups. Furthermore, the $\mathrm{Sm} / \mathrm{TS}$ ratio was significantly greater in the high-risk $S$. mutans and non-detectable $S$. sobrinus subgroups of the CA group. Additionally, the $\mathrm{Sm} / \mathrm{TS}$ ratio was significantly greater in the high-risk $\mathrm{Sm} / \mathrm{TS}$ plus detectable $S$. sobrinus subgroup than in the low-risk $\mathrm{Sm} / \mathrm{TS}$ plus detectable $S$. sobrinus subgroup, thereby confirming the usefulness of the Sm/TS ratio. Larger studies should be performed in the future to prospectively investigate the relationship between caries activity and the onset of caries formation. The results of this study confirmed the value of quantitative plaque assessment and caries ratio assay. Based on these initial results, a simple assay that involves culture for detecting $S$. mutans and $S$. sobrinus without immunohistochemical analysis or PCR is being developed.

\section{Conclusion}

The results of this study showed that the number of DMF teeth was greater in plaque samples of adults with concomitant infections of $S$. mutans (high levels) and $S$. sobrinus as compared with that in a single infection. These findings indicate the usefulness of analysis of cariogenic dental bacteria to detect adult populations with the severity of dental caries according to the $\mathrm{Sm} / \mathrm{TS}$ ratio using dental plaque samples.

\section{Competing Interests}

The authors have no competing interest to declare.

\section{Acknowledgement}

Not applicable 
Citation: Gotouda H, Shinozaki-Kuwahara N,Taguchi C, Ohta M, Shimosaka M, et al. (2017) Evaluation of Dental Caries and Concomitant Infection Based on the Ratio of Cariogenic Bacteria using Plaque Samples in Adults. Dentistry 7: 461. doi:10.4172/2161-1122.1000461

Page 5 of 5

\section{Funding}

This study was self-funded by authors.

\section{References}

1. Axelsson P (2000) Diagnosis and risk prediction of dental caries. Illinois: Quintessence, pp: 1-120.

2. Guo L, Shi W (2013) Salivary biomarkers for caries risk assessment. J Calif Dent Assoc 41: 107-118.

3. Maheswari SU, Raja J, Kumar A, Seelan RG (2015) Caries management by risk assessment: A review on current strategies for caries prevention and management. J Pharm Bioallied Sci 7: S320-2S324.

4. Parisotto TM, Steiner-Oliveira C, Silva CM, Rodrigues LK, Nobre-dosSantos M (2010) Early childhood caries and mutans streptococci: a systematic review. Oral Health Prev Dent 8: 59-70.

5. D'Amario M, Barone A, Marzo G, Giannoni M (2006) Caries-risk assessment: the role of salivary tests. Minerva Stomatol 55: 449-463.

6. Okada M, Soda Y, Hayashi F, Doi T, Suzuki J, et al. (2005) Longitudinal study of dental caries incidence associated with Streptococcus mutans and Streptococcus sobrinus in pre-school children. J Med Microbiol 54: 661-665.

7. Sanchez-Acedo M, Montiel-Company JM, Dasi-Fernandez F, AlmerichSilla JM (2013) Streptococcus mutans and Streptococcus sobrinus detection by polymerase chain reaction and their relation to dental caries in 12 and 15 year-old schoolchildren in Valencia (Spain). Med Oral Patol Oral Cir Bucal 18: 839-845.

8. de Soet JJ, van Loveren C, Lammens AJ, Pavicic MJ, Homburg CH, et al (1991) Differences in cariogenicity between fresh isolates of Streptococcus sobrinus and Streptococcus mutans. Caries Res 25: 116-122.

9. Okada M, Kawamura M, Oda Y, Yasuda R, Kojima T, et al. (2012) Caries prevalence associated with Streptococcus mutans and Streptococcus sobrinus in Japanese schoolchildren. Int J Paediatr Dent 22: 342-348.

10. Okada M, Taniguchi Y, Hayashi F, Doi T, Suzuki J, et al. (2010) Late established mutans streptococci in children over 3 years old. Int J Dent 2010: 732468

11. Hedge PP, Ashok BR, Ankola VA (2005) Dental caries experience and salivary levels of Streptococcus mutans and lactobacilli in 13-15 years old children of Belgaum city, Karnataka. J Indian Soc Pedo Prev Dent 23: 23-26.

12. Loyola-Rodriguez JP, Martinez-Martinez RE, Flores-Ferreira BI, Pati-oMarÃn N, Alpuche-SolÃs AG, et al. (2008) Distribution of Streptococcus mutans and Streptococcus sobrinus in saliva of Mexican preschool cariesfree and caries-active children by microbial and molecular (PCR) assays. J Clin Pediatr Dent 32: 121-126.

13. Kishi M, Abe A, Kishi K, Ohara-Nemoto Y, Kimura S, Yonemitsu M (2009) Relationship of quantitative salivary levels of Streptococcus mutans and Streptococcus sobrinus in mothers to caries status and colonization of mutans streptococci in plaque in their 2.5-year-old children. Community Dent Oral Epidermiol 37: 241-249.
14. Singh N, Chawla HS, Tewari A, Sachdev V (2010) Correlation of severity of streptococcus mutans in the saliva of school children of Chandigarh with dental caries. BFUDJ; 7: 5-8.

15. Nurelhuda NM, Al-Haroni M, Trovik TA, Bakken V (2010) Caries experience and quantification of streptococcus mutans and streptococcus sobrinus in saliva of sudanese school children. Caries Res 44: 402-407.

16. Nanda J, Sachdev V, Sandhu M, Deep-Singh-Nanda K (2015) Correlation between dental caries experience and mutans streptococci counts using saliva and plaque as microbial risk indicators in 3-8 year old children. A cross sectional study. J Clin Exp Dent 7: e114-e118.

17. Batoni G, Ota F, Gheraldi S, Senesi S, Barnini S, et al. (1992) Epidemiological survey of Streptococcus mutans in a group of adult patients living in Pisa (Italy). Eur J Epidemiol 8: 238-242.

18. Nishikawara F, Katsumura S, Ando A, Tamaki Y, Nakamura Y, et al. (2006) Correlation of cariogenic bacteria and dental caries in adults. J Oral Sci 48: 245-251.

19. Li Y, Tanner A (2015) Effect of antimicrobial intervention on oral microbiota associated with early childhood caries. Pediatr Dent 37: 226-244.

20. Neta T, Inokuchi R, Shinozaki-Kuwahara N, Kouno Y, Ikemi T, et al. (2002) Investigation of microbiological methods to estimating individual caries risk: evaluation of sampling methods and materials. Int J Oral-Med Sci 1: 29-32.

21. Hsu KLC, Osgood RC, Cutter GR, Childers NK (2010) Variability of two plaque sampling methods in quantitation of Streptococcus mutans. Caries Res 44: 160-164.

22. Ghasempour M, Rajabnia R, Irannejad A, Hamzeh M, Ferdosi E, et al (2013) Frequency, biofilm formation and acid susceptibility of streptococcus mutans and streptococcus sobrinus in saliva of preschool children with different levels of caries activity. Dent Res J (Isfahan); 10: 440-445.

23. Gold OG, Jordan HV, Houte JV (1973) A selective medium for Streptococcus mutans. Arch Oral Biol 18: 1357-1364.

24. World Health Organization (1997) Oral health surveys: basic methods, (4th edn.), World Health Organization, Geneva, Switzerland.

25. da Silva Bastos Vde A, Freitas-Fernandes LB, Fidalgo TK, Martins C, Mattos CT, et al. (2015) Mother-to-child transmission of Streptococcus mutans: a systematic review and meta-analysis. J Dent 43: 181-191.

26. Marsh PD (2003) Are dental diseases examples of ecological catastrophes? Microbiology 149: 279-294.

27. Loesche WJ (1986) Role of Streptococcus mutans in human dental decay. Microbiol Rev 50: 353-380.

28. Köhler B, Bjarnason S (1992) Mutans Streptococci, lactobacilli and caries prevalence in 15 to 16-year olds in Goteborg. Part II. Swed Dent J 16: 253-259.

29. Hamada S, Slade HD (1980) Biology, immunology, and cariogenicity of Streptococcus mutans. Microbiol Rev 44: 331-384. 